\title{
Capital social y salud en América Latina y el Caribe: una revisión sistemática
}

\author{
Cristóbal E. Kripper y Jaime C. Sapag ${ }^{1}$
}

Forma de citar Kripper CE, Sapag JC. Capital social y salud en América Latina y el Caribe: una revisión sistemática. Rev Panam Salud Publica. 2009;25(2):162-70.

RESUMEN Objetivo. Identificar la información validada disponible sobre la relación entre el capital social (CS) y la salud en América Latina y el Caribe (ALC).

Métodos. Se realizó una búsqueda sistemática de los trabajos publicados entre enero de 1990 y junio de 2007 en las bases de datos Medline, SciELO, LILACS y The Cochrane Library. Se analizaron todos los artículos de investigación y revisión publicados en revistas científicas, que evaluaran el CS y su relación con la salud en ALC.

Resultados. Se encontraron 15 artículos (11 de investigación original y 4 de revisión). Las áreas de salud exploradas por las investigaciones originales fueron: a) mortalidad y esperanza de vida, b) salud mental, c) traumas, d) estado nutricional y e) vacunación. Se analiza detalladamente la validez de estos trabajos, sus resultados y principales conclusiones.

Conclusiones. A pesar de las limitaciones propias del concepto de CS y de los estudios identificados, se puede afirmar que existe evidencia científica incipiente de la posible relación entre el CS y la salud en ALC. El CS podría desempeñar un papel protector en ciertas áreas sanitarias, como la prevención de traumas y la salud mental; no obstante, la relación entre el CS y la salud podría variar según los subtipos de CS y los contextos socioeconómicos y culturales específicos. Resulta fundamental continuar desarrollando investigaciones en el ámbito de la epidemiología social, en las que se analice en profundidad la relación entre los determinantes sociales y los aspectos específicos de la salud en el contexto de ALC.

Palabras clave Participación social, salud, América Latina, Región del Caribe.

Cada vez es más evidente la importancia de los determinantes sociales para enfrentar los desafíos actuales de la salud y el desarrollo en el mundo. Estos determinantes son las características específicas y las vías por las cuales las condiciones de la sociedad influyen en la salud $(1,2)$. El tipo y la calidad de las relaciones sociales, la cultura, el barrio de residencia, el trabajo, el nivel socioeconómico, así como la pertenencia étnico-racial y el género,

1 Departamento de Medicina Familiar, Pontificia Universidad Católica de Chile, Santiago, Chile. La correspondencia se debe dirigir a Jaime C. Sapag, Departamento de Medicina Familiar, Pontificia Universidad Católica de Chile, Calle Lira No. 40, Santiago Centro, Chile. Correos electrónicos: jsapag@med.puc.cl, jsapagm@yahoo.es entre otras variables, han sido frecuente objeto de estudio en investigaciones relacionadas con la salud (3). Es una obligación ética desarrollar intervenciones eficaces en el ámbito de la salud desde una perspectiva más amplia que aborde adecuadamente los determinantes sociales y contribuya a la equidad en salud.

En los últimos años se ha descrito la importancia que puede tener el capital social (CS) en el campo de la salud pública, así como en materias tan diversas como el desarrollo social, la superación de la pobreza (4), la disminución de la criminalidad (5) y el fortalecimiento de la educación (6).

No existe una definición única de CS, como tampoco hay una sola clasificación de sus formas o tipos (7-8). Por ejemplo, Bourdieu y Loïc afirman que el CS es el conjunto de recursos reales o potenciales que se vinculan con la posesión de una red duradera de relaciones más o menos institucionalizadas de conocimiento o reconocimiento mutuo (9). Sin embargo, Coleman pone el énfasis en los elementos de la estructura social que facilitan ciertas acciones de los actores dentro de dicha estructura (10). Por su parte, para Putnam y colaboradores, el concepto de CS abarca determinados elementos de la organización social —como la confianza, las normas y las redes sociales- que pueden mejorar la eficiencia de la sociedad al facilitar la acción coordinada (11). Sin embargo, más allá de la variedad de 
definiciones, hay cierto consenso en considerar que se trata de un recurso intangible y dinámico que existe en el colectivo y abarca elementos como la confianza, la participación y la reciprocidad (7-12).

Se reconocen dos tipos de CS: a) el CS cognitivo, que se refiere a ideas y procesos mentales reforzados por la cultura, la ideología, las normas, los valores, las actitudes y las creencias que contribuyen al comportamiento corporativo; y b) el CS estructural, que está más relacionado con los aspectos observables de la organización social, como el papel desempeñado por cada actor, las reglas, los precedentes, los procedimientos y la amplia variedad de redes que contribuyen a la cooperación. En el CS estructural se pueden distinguir dos dimensiones relacionales: la de tipo vertical, en la cual los grupos que interactúan tienen distintos niveles de poder y recursos —algo de suma importancia en el proceso de definición e implementación de políticas-y la de tipo horizontal, donde las relaciones se dan entre grupos que comparten características similares -demográficas o no-, también relevantes en los procesos de participación cívica, la construcción de coaliciones, etc. (13).

Diversos estudios indican que el CS desempeñaría un papel positivo en la salud. Las comunidades con mayor CS presentarían una mayor expectativa de vida y una mejor percepción de su salud, así como menores tasas de morbilidad y mortalidad por causas generales y específicas, incluso después de ajustar por el nivel socioeconómico y educacional y otras variables (14-17). Sin embargo, no siempre los datos científicos son claros, por lo que algunos autores se muestran más escépticos con respecto a las posibilidades de aplicación de este concepto en la salud $(18,19)$. Otros estudios indican que con relación a la salud, el CS puede desempeñar un papel positivo en algunos casos y negativo en otros (20). Todo lo anterior conlleva interesantes desafíos para fortalecer las metodologías de investigación y las intervenciones en salud en todo el mundo, particularmente en América Latina y el Caribe (ALC) $(21,22)$.

Si bien el CS ha surgido como un atractivo concepto y un posible aporte en el campo de la salud pública, el desarrollo teórico al respecto se ha establecido principalmente a partir de investigaciones originadas en los llamados países desarrollados, con realidades socioculturales muy distintas a las de ALC. Por ello, sus resultados se deben evaluar con prudencia al momento de buscar su aplicabilidad en el contexto latinoamericano y caribeño, más aún si se toman en cuenta las disímiles interpretaciones de los conceptos empleados para medir el CS en diferentes sociedades (23). La aceptación del CS como herramienta que influye en el desarrollo de la salud pública en ALC requiere implementar medidas apoyadas en resultados generados también localmente. Sin embargo, no se conoce ningún análisis estructurado llevado a cabo en ALC sobre el CS y su relación con la salud hasta la fecha.

La presente revisión tiene por objetivo identificar la información validada disponible sobre la relación entre el CS y la salud, específicamente en el contexto de ALC. Se pretende, además, contribuir a identificar desafíos futuros para la investigación en este campo en ALC.

\section{MATERIALES Y MÉTODOS}

Se realizó una búsqueda sistemática de los trabajos publicados entre enero de 1990 y junio de 2007 en las bases de datos Medline, SciELO, LILACS y The Cochrane Library. Como motores de búsqueda se utilizaron los términos "capital social" y "salud" combinados con "América Latina" y cada uno de los nombres de los países de ALC.

Se analizaron todos los artículos de investigación y revisión publicados en revistas científicas, que evaluaran explícitamente el CS y que exploraran su relación con alguna variable de salud bien definida en el ámbito de ALC, independientemente del idioma de publicación.

Los autores seleccionaron de forma independiente los estudios que presentaran evidencias sobre la posible relación entre el CS y la salud en el contexto de ALC y, posteriormente, realizaron una valoración crítica de cada uno para esta revisión.

\section{RESULTADOS}

El incipiente desarrollo en el conocimiento sobre la relación entre el CS y la salud en ALC se manifiesta en el hecho de que casi todos los estudios encontrados se publicaron en los últimos dos años. La búsqueda dio como resultado un total de 15 artículos, de los cuales 11 son de investigación original y 4 de revisión. Medline resultó ser el motor de búsqueda más eficaz, ya que solo uno de los artículos de revisión mencionados es- capó a su alcance. Las principales características de los estudios originales se resumen en el cuadro 1.

Las cinco áreas de salud a las que se dirigían las investigaciones originales sobre el CS y aspectos de salud en ALC fueron: a) mortalidad y esperanza de vida, b) salud mental, c) traumas, d) estado nutricional y e) vacunación. No se encontraron estudios tendientes a evaluar otras causas específicas de morbimortalidad. Los artículos originales revisados provenían de seis países de América Latina (Bolivia, Brasil, Colombia, México, Nicaragua y Perú).

Debido a la escasez de publicaciones científicas sobre la relación entre el CS y la salud en ALC se tomaron en cuenta para el análisis todos los artículos encontrados y se valoró críticamente su calidad científica. A continuación se presenta un resumen de los artículos de investigación original, por constituir un reflejo más sólido del estado actual de la experiencia latinoamericana en relación con el CS y la salud. En la discusión se incorporan al análisis los artículos de revisión encontrados.

\section{Mortalidad y esperanza de vida}

Dos estudios transversales, uno de México (24) y otro de Colombia (25), evaluaron la relación entre el CS y la mortalidad o la esperanza de vida. Ambos definieron el CS, bajo una perspectiva ecológica, como las características de la organización social —redes de asociaciones secundarias, niveles altos de confianza entre las personas y normas de ayuda mutua y reciprocidad- que se manifiestan como recursos para las personas y facilitan su acción colectiva.

En el artículo mexicano (24) se analizó si el efecto contextual potencial que tiene la desigualdad en los ingresos sobre la esperanza de vida al nacer se encuentra mediado por la corrupción ajustada por diferencias culturales (por ejemplo, la proporción de la población que profesa la religión católica o que habla lengua indígena, como indicadores indirectos de esos factores) como un reflejo del CS. Para ello se realizó un análisis estadístico (correlación de Spearman y regresiones lineales múltiples) con los datos de la esperanza de vida al nacer, el coeficiente de Gini para la desigualdad en los ingresos y el índice de corrupción y buen gobierno de los 32 estados federativos de México. El principal resultado de este estudio fue 
CUADRO 1. Características de los estudios sobre capital social (CS) y salud en América Latina y el Caribe incluidos en la revisión sistemática

\begin{tabular}{|c|c|c|c|c|c|}
\hline País & Diseño & Población & $\begin{array}{l}\text { Resultado en } \\
\text { salud evaluado }\end{array}$ & $\begin{array}{l}\text { Instrumentos empleados y } \\
\text { parámetros evaluados }\end{array}$ & Ref. \\
\hline Bolivia & Transversal cuantitativo & $\begin{array}{l}\text { Adultos de } 13 \text { villas indígenas } \\
\text { preindustriales de la Amazonia } \\
\text { boliviana }(n=655)\end{array}$ & $\begin{array}{l}\text { Emociones negativas } \\
\text { Consumo de alcohol }\end{array}$ & $\begin{array}{l}\text { CS: regalos y trabajo comunitario } \\
\text { Salud mental: tristeza, ira, miedo } \\
\text { Alcoholismo: consumo semanal }\end{array}$ & 38 \\
\hline Bolivia & Transversal cuantitativo & $\begin{array}{l}\text { Jefes de hogar y mayores de } \\
13 \text { años }(n=479)\end{array}$ & Estado nutricional & $\begin{array}{l}\text { CS: regalos y trabajo comunitario } \\
\text { Estado nutricional: índice de masa corporal, pliegues } \\
\text { cutáneos tricipital y subescapular y el área muscular } \\
\text { braquial } \\
\text { Desigualdad en los ingresos: coeficiente de Gini para } \\
\text { ingresos per cápita y área de deforestación alrededor } \\
\text { de la vivienda }\end{array}$ & 50 \\
\hline Brasil & Transversal cuantitativo & $\begin{array}{l}\text { Adolescentes de } 14-15 \text { años, } \\
\text { mayoritariamente de nivel socio- } \\
\text { económico medio ( } n=1302)\end{array}$ & $\begin{array}{l}\text { Daños dentales de origen } \\
\text { traumático }\end{array}$ & $\begin{array}{l}\text { CS: instrumento de } 30 \text { preguntas elaborado por los } \\
\text { autores; resultados agregados a nivel de vecindario } \\
\text { Daño dental: evaluación física estandarizada }\end{array}$ & 44 \\
\hline Colombia & Transversal cuantitativo & 33 departamentos de Colombia & Mortalidad por cáncer & $\begin{array}{l}\text { CS: tasa de muertes violentas en 1973-1996 por } \\
100000 \text { habitantes (como indicador inverso del CS) } \\
\text { Mortalidad por cáncer: tasa acumulada en 1990-1996 } \\
\text { por } 100000 \text { habitantes }\end{array}$ & 25 \\
\hline Colombia & $\begin{array}{l}\text { Transversal cualitativo y } \\
\text { cuantitativo }\end{array}$ & $\begin{array}{l}\text { Personas de } 15 \text { a } 25 \text { años de } \\
\text { bajo nivel socioeconómico } \\
(n=1057)\end{array}$ & $\begin{array}{l}\text { Depresión y ansiedad } \\
\text { Alcoholismo } \\
\text { Violencia }\end{array}$ & $\begin{array}{l}\text { CS: instrumento adaptado para la evaluación del CS (31) } \\
\text { Salud mental: cuestionario de autoinforme (32) } \\
\text { Alcoholismo: cuestionario CAGE (34) } \\
\text { Violencia: cuestionario del Proyecto ACTIVA (33) }\end{array}$ & 30 \\
\hline Colombia & Transversal cuantitativo & $\begin{array}{l}\text { Personas de } 15 \text { a } 25 \text { años de } \\
\text { bajo nivel socioeconómico } \\
(n=1168)\end{array}$ & $\begin{array}{l}\text { Depresión y ansiedad } \\
\text { Violencia }\end{array}$ & $\begin{array}{l}\text { CS: instrumento adaptado para la evaluación del CS (31) } \\
\text { Salud mental: cuestionario de autoinforme (32) } \\
\text { Alcoholismo: cuestionario del Proyecto ACTIVA (33) }\end{array}$ & 29 \\
\hline México & Transversal cuantitativo & 32 estados federales & Esperanza de vida & $\begin{array}{l}\text { CS: índice de corrupción y buen gobierno, } 2001 \\
\text { Desigualdad en los ingresos: coeficiente de Gini } \\
\text { Esperanza de vida al nacer }\end{array}$ & 24 \\
\hline México & Transversal cuantitativo & $\begin{array}{l}\text { Personas de tres zonas con } \\
\text { diferentes tasas de accidentes } \\
\text { de tránsito }(n=323)\end{array}$ & Accidentes de tránsito & $\begin{array}{l}\text { CS: cuestionario elaborado por los autores } \\
\text { Accidentes de tránsito: percepción de los accidentes } \\
\text { como problema local }\end{array}$ & 46 \\
\hline Nicaragua & Transversal cuantitativo & $\begin{array}{l}\text { Hogares pertenecientes a seis } \\
\text { villas semiurbanas o rurales } \\
(n=482)\end{array}$ & Vacunación infantil & $\begin{array}{l}\text { CS: cuestionario integrado para la medición del CS (53) } \\
\text { Vacunación infantil: puntuación según el numero de } \\
\text { vacunas recibidas }\end{array}$ & 52 \\
\hline Perúa & $\begin{array}{l}\text { Transversal cuantitativo } \\
\text { (niveles individual y } \\
\text { comunitario) }\end{array}$ & $n=1678$ & Estado nutricional infantil & $\begin{array}{l}\text { CS: versión resumida del instrumento modificado para } \\
\text { la evaluación del CS } \\
\text { Estado nutricional infantil: índices } Z \text { del peso para la } \\
\text { edad y la talla para la edad }\end{array}$ & 51 \\
\hline Perúa & $\begin{array}{l}\text { Transversal cuantitativo } \\
\text { (niveles individual y } \\
\text { comunitario) }\end{array}$ & $n=1659$ & $\begin{array}{l}\text { Depresión y ansiedad } \\
\text { maternas }\end{array}$ & $\begin{array}{l}\text { CS: versión resumida de un instrumento modificado } \\
\text { para la evaluación del CS (23) } \\
\text { Salud mental materna: cuestionario de autoinforme (43) }\end{array}$ & 41 \\
\hline
\end{tabular}

aA partir de un estudio más amplio realizado en Etiopía, India, Perú y Vietnam (42).

demostrar el efecto negativo que ejerce la desigualdad en los ingresos sobre la esperanza de vida al nacer. Igualmente se observó una correlación significativa entre el índice de corrupción y buen gobierno y la esperanza de vida al nacer (coeficiente de Spearman $=-0,3592 ; P<0,05)$. En ese artículo se plantea que la relación entre la desigualdad en los ingresos y la esperanza de vida al nacer podría estar mediada por el CS, expresado en los niveles de corrupción y las diferencias culturales.
Es importante, sin embargo, señalar algunas limitaciones de ese estudio: a) no se evaluó directamente el CS; b) no se ajustaron los modelos evaluados por otras variables importantes; c) las variables culturales elegidas (legado indígena 
y religión católica) tienen una relevancia particular en el contexto mexicano, pero no necesariamente pueden generalizarse a otros países de la Región; y d) no se expuso claramente por qué la corrupción ajustada por diferencias culturales puede ser un buen indicador del CS.

En el estudio colombiano (25), el mismo autor evaluó la relación entre el CS y la tasa de mortalidad por cáncer. Esta vez, su aproximación al CS se basó en la teoría del CS "perverso" (en oposición al CS "productivo"); así la variable sustituta (también llamada proxy) para evaluar el CS fue la tasa de muertes violentas, ya que según esta teoría, mayores tasas de muertes violentas serían un reflejo de mayores niveles de CS "perverso". Se determinó la tasa acumulada de mortalidad por cáncer entre 1990 y 1996 y su correlación con la tasa de muertes violentas ocurridas entre 1973 y 1996. Para ello se tomó esta última como indicador directo y se ajustaron los resultados por la convergencia/polarización económica y la razón de desplazamiento interno en los 32 departamentos del país y el distrito capital, Bogotá. El análisis estadístico mostró que a mayor tasa de muertes violentas existe una tasa de mortalidad por cáncer significativamente mayor. Es importante analizar estos resultados ya que en estudios previos realizados en Colombia se observó una relación entre la violencia y el CS. Algunos autores han sugerido que los efectos adversos del CS estarían relacionados con los comportamientos coercitivos y conservadores de las sociedades cohesionadas, que sobrecargan a sus miembros con obligaciones y desalientan las conductas que promueven la salud, mientras que los efectos positivos se asociarían con las redes secundarias, los altos niveles de confianza entre las personas y las normas de ayuda mutua y reciprocidad (26-28).

Ambas investigaciones corresponden a aproximaciones de carácter transversal y utilizan una estimación indirecta y poco habitual del CS, por lo que sus hallazgos deben ser tomados con cautela. Estos resultados, no obstante, constituyen un paso de avance importante en este campo para estos países y la Región y subrayan la importancia de entender el CS en cada contexto como un recurso que puede expresarse favorable o desfavorablemente.

\section{Salud mental}

Se encontraron cuatro estudios sobre la relación entre el CS y la salud mental en tres países de ALC: Colombia, Bolivia y Perú.

Colombia. Dos de los estudios realizados por Harpham y colaboradores se basaron en una encuesta aplicada en el año 2002 en una población joven de dos barrios de Aguablanca, zona de bajo nivel socioeconómico de la ciudad de Cali, Colombia, reconocida además por sus altos niveles de violencia $(29,30)$. Esta investigación formó parte de un estudio-intervención cuasiexperimental. Los autores definieron el CS como el conjunto de inversiones en recursos y redes que producen cohesión social, confianza y voluntad de participar en actividades comunitarias. El CS se estimó mediante una versión adaptada de un instrumento de evaluación de CS (31) con el fin de medir la percepción y la experiencia individual más que de evaluar el CS a nivel ecológico. Los componentes evaluados por la encuesta fueron: la participación, la confianza, la cohesión social, el apoyo social y el control social no formal.

El primero de esos artículos (29) es un estudio transversal cuantitativo realizado en 1168 jóvenes colombianos de 15 a 25 años y tuvo por objetivo determinar si existía una asociación independiente entre el CS y la salud mental a partir de algunas variables demográficas y los niveles de violencia social. En cuanto a la salud mental, el estudio consideró la prevalencia de probables casos de depresión y ansiedad mediante el cuestionario de autoinforme recomendado por la Organización Mundial de la Salud en 2001 (32) y la ideación suicida; los niveles de violencia fueron evaluados mediante el cuestionario desarrollado por el Proyecto ACTIVA (33). En esta investigación se encontró una población socialmente fragmentada, con altos niveles de pobreza y bajos niveles de religiosidad. El nivel de confianza hacia la población en general fue el único componente del CS que se correlacionó con la salud mental, aunque con una significación estadística cercana al límite, de modo que a mayores niveles de confianza menor fue la probabilidad de padecer ansiedad y depresión (razón de posibilidades [odds ratio, OR] $=0,8$; intervalo de confianza de 95\% [IC95\%]: 0,7 a 1,$0 ; P<0,044)$. Al incluir el nivel de violencia en el modelo, la asociación entre el CS y la salud mental perdió su significación y los principales factores de riesgo fueron el ser del sexo femenino $(\mathrm{OR}=4,3)$, la baja escolaridad $(\mathrm{OR}=2,7)$, la violencia intrafamiliar $(\mathrm{OR}=1,7)$, el ser víctima de la violencia $(\mathrm{OR}=1,6)$, la percepción de violencia en la comunidad $(\mathrm{OR}=1,5)$ y el presentar conductas violentas $(\mathrm{OR}=1,4)$. Pese a incluir comentarios sobre los resultados cualitativos, en el artículo no se describe con mayor detalle la metodología utilizada.

El segundo artículo de este grupo (30), publicado en 2005 a partir de un estudio cuantitativo y cualitativo, complementó los resultados anteriores con la evaluación del consumo de bebidas alcohólicas mediante el instrumento conocido como CAGE (34) En este artículo se confirma lo observado anteriormente con respecto al CS y la salud mental. Los autores mencionaron la ideación suicida en su análisis, pero no se describen los resultados encontrados. Aunque el alcoholismo fue un factor de riesgo de ansiedad y depresión, la significación estadística de este resultado se perdió al incluir en el análisis el CS y la violencia. El componente cualitativo del estudio, centrado en la discusión sobre el CS y los niveles de violencia, se llevó a cabo en el año 2004 en 16 grupos focales compuestos por jóvenes de 15 a 25 años. Se confirmó la fragmentación social descrita en el estudio cuantitativo, los bajos niveles de confianza en los residentes de la comunidad en general y la mayor confianza en los vecinos cercanos. De acuerdo con los grupos focales, la pobreza fue el principal factor asociado con la tensión en los ámbitos público y doméstico.

Según este artículo, se observaron avances en la validación del instrumento elaborado para medir el CS. Al aplicar de nuevo el cuestionario a 5\% de la muestra con nuevos entrevistadores se obtuvo un coeficiente de correlación $r>0,7$ en $67 \%$ de los tópicos evaluados. También se debe destacar que la incorporación de la metodología cualitativa amplió las posibilidades del estudio, ya que permitió explorar adecuadamente el contexto subyacente.

Entre las limitaciones de esta investigación se pueden mencionar: a) se analizó una sola comunidad o área geográfica de Cali; b) por su naturaleza transversal no se pudo inferir causalidad; y c) se debe considerar la posible causalidad inversa como problema, pues la presencia de depresión o ansiedad pudo haber afectado la percepción que algunos participantes tenían del contexto. Además, el diseño estadístico de la segunda investigación no consideró el CS como un factor protector o de riesgo del alcoholismo, algo que se refleja 
en la literatura científica internacional con resultados contradictorios (35-37).

Bolivia. Se encontró un estudio transversal cuantitativo realizado en 655 indígenas de la selva amazónica en Bolivia (38). Se entendió por CS la confianza, las redes sociales y las organizaciones comunitarias que permiten actuar colectivamente. El CS colectivo se infirió a partir del CS individual medido mediante una encuesta aplicada a los participantes sobre su conducta durante los últimos siete días en relación con regalos que hubieran entregado, el número de veces que ofrecieron ayuda en un trabajo o que realizaron labores comunitarias. Como variables dependientes se consideraron el consumo de alcohol y el sentimiento de emociones negativas (ira, tristeza o miedo) durante los últimos siete días. En los modelos de regresión multifactoriales, el CS colectivo en la villa se comportó como un factor protector, ya que se asoció significativamente con un menor consumo de bebidas alcohólicas y una menor frecuencia de emociones negativas. Por ejemplo, el incremento en un episodio semanal de CS en una villa se asoció con un consumo de alcohol entre 0,22 y 0,44 veces menor $(P<0,009)$.

Explorar las emociones negativas y la percepción de salud emocional sin una definición clínica, como en otras investigaciones (39), es una limitación de este estudio, especialmente si se toma en cuenta que la amplitud de estos conceptos dificulta la valoración de los resultados. Es importante destacar, además, que el período de siete días utilizado para explorar el consumo de bebidas alcohólicas puede afectar al autoinforme (40).

Perú. En el cuarto estudio encontrado sobre el CS y la salud mental (41), los autores utilizaron los resultados del estudio internacional sobre la juventud (42) realizado en el marco del proyecto Young Lives Study. ${ }^{2}$ En este estudio se exploraron los CS cognitivo y estructural mediante una metodología transversal cuantitativa a partir de una versión resumida del instrumento modificado para la evaluación in-

\footnotetext{
2 Young Lives: an International Study of Childhood Poverty es un proyecto de investigación a largo plazo que se realiza desde 2002 en cuatro países -Etiopía, India, Perú y Vietnam - dirigido a identificar las causas y las consecuencias de la pobreza infantil e informar las políticas dirigidas a reducir la pobreza. Para más información visitar http:// www.young lives.org.uk/
}

dividual del CS (Adapted Social Capital Assessment Tool, ASCAT) (23). El CS comunitario se estimó mediante la agregación de los valores de CS individual y se analizó la asociación de ambos con la depresión y la ansiedad materna mediante el cuestionario de autoinforme SRQ-20 (43). En este caso, la definición de CS abarcó las normas, las redes y las asociaciones que facilitan la acción colectiva, con una visión integradora que reconoce su valor tanto a escala individual como ecológica. Su exploración comprendió la membresía a grupos, el apoyo social, el rol cívico y el CS cognitivo (confianza, armonía social, percepción de justicia y sentido de pertenencia). En comparación con Vietnam, Etiopía y la India, en Perú $(n=1$ 659) se encontraron los niveles más bajos de CS en las cuatro áreas mencionadas. Al igual que en los otros tres países, el CS cognitivo individual en la muestra peruana se asoció con menos problemas de salud mental materna (OR ajustado =0,61; IC95\%: 0,48 a 0,78$)$. En cambio, los resultados relacionados con el CS estructural variaron más de un país a otro. Por ejemplo, en Perú se observó que las madres con mayores niveles de apoyo social individual presentaban mayor prevalencia de ansiedad y depresión (OR ajustado = 1,39; IC95\%: 1,02 a 1,88).

Si bien este estudio tiene las limitaciones propias del diseño transversal y el sesgo generado por la agregación de los resultados individuales para interpretar la situación del colectivo, en él se demuestra el papel protector que desempeñaría el CS cognitivo en la salud mental y se refuerza la relación hallada en el estudio colombiano realizado en Cali (29, 30). Sin embargo, se observó también que algunos componentes del CS pueden comportarse como factores de riesgo en ciertos contextos. Este grupo de investigadores contribuyó, además, con un importante avance en la metodología de la investigación en ALC al profundizar en el proceso de validación de su instrumento de medición del CS (23).

\section{Traumas}

Una reciente investigación transversal cuantitativa exploró en 1302 adolescentes la prevalencia de daños dentales de origen traumático ${ }^{3}$ y su asociación con el

\footnotetext{
Se refiere a los daños provocados a alguno de los cuatro dientes anteriores - superiores o inferiores- por fracturas o avulsiones traumáticas, evaluados mediante el examen de la cavidad oral.
}

CS en las ciudades satélite Taguatinga y Ceilândia, ubicadas en el Distrito Federal, Brasil (44).

El CS se definió como las normas y las redes que permiten el trabajo colectivo. Se diseñó un instrumento dirigido a los padres para evaluar la confianza social, el control social, el empoderamiento, la seguridad vecinal y la eficacia política. Mediante subescalas (se aplicó el análisis de componentes principales), los autores generaron un índice de CS promedio representativo para cada uno de los 37 vecindarios de origen. Los valores del coeficiente kappa para el examen de diagnóstico por duplicado fueron superiores a 0,92 .

La prevalencia de daños dentales de origen traumático en la población masculina fue significativamente menor en las zonas con mayor CS. El hogar y la calle fueron los lugares donde ocurrieron estos traumas con mayor frecuencia (44,3\% y $26,7 \%$, respectivamente). Después de ajustar por algunas variables individuales y vecinales, el OR ajustado para el incremento de una unidad en el índice estandarizado del CS fue de $0,55(P<0,05)$ en hombres. No hubo resultados significativos en la población femenina.

Al interpretar los resultados de este estudio se deben tomar en cuenta las consideraciones propias del diseño transversal, el sesgo de agregación y las limitaciones de la validación del instrumento utilizado para medir el CS, ya que no se tomó en cuenta la membresía a grupos y la reciprocidad. Además, la ambigüedad de la definición de "barrio" y las percepciones de los entrevistados en torno a sus límites impidieron saber con exactitud cuándo las respuestas se referían al barrio en sí mismo (según la definición de los investigadores) y cuándo al entorno más inmediato percibido por los participantes (una o dos calles más próximas) (45).

Sin embargo, los autores realizaron un notable aporte al mostrar de manera más objetiva y comparable el nivel de CS (mediante el índice estandarizado de CS) de la unidad evaluada y su relación con una situación de salud específica: los daños dentales de origen traumático.

En otro estudio, Inclán y colaboradores (46) analizan la relación entre el CS y los accidentes de tránsito en Cuernavaca, capital del Estado de Morelos, México. Se utilizó como definición de CS la capacidad de los miembros de una comunidad de 
trabajar juntos en favor de metas comunes basadas en la confianza social, mediante redes sociales y normas que promuevan la coordinación y la cooperación para beneficio mutuo. En una muestra de 323 personas procedentes de tres zonas con diferentes tasas de accidentes de tránsito se evaluó el CS mediante una encuesta elaborada por los autores para indagar sobre la participación de los entrevistados en organizaciones locales, sus expectativas respecto al papel que deben desempeñar los vecinos para incrementar la seguridad en el tránsito, la influencia colectiva en la seguridad vial, el respeto de las normas de tránsito (como reflejo de reciprocidad) y los signos de desorganización a partir de la percepción del espacio físico y la seguridad individual. El análisis de los modelos multifactoriales ajustados mostró que el tener bajas expectativas con respecto al papel de los vecinos en el incremento de la seguridad -entendido por los autores como un bajo CS- presentó una correlación estadísticamente significativa con el hecho de identificar los accidentes de vehículos motorizados como un problema de la comunidad (OR ajustado $=2,02$; IC95\%: 1,14 a 3,67).

Es importante señalar que en este estudio no se encontró una relación entre el CS y los accidentes directamente. La encuesta utilizada evaluó el CS a partir de las expectativas de los entrevistados, lo que no reflejó necesariamente la realidad, sino las esperanzas comunes sobre ella. Es necesario interpretar cuidadosamente estos resultados.

\section{Estado nutricional}

Diversos autores han mostrado la correlación existente entre la desigualdad en los ingresos económicos y la salud $(47,48)$ y se discute el papel que podría desempañar el CS en dicha asociación (49). Godoy y colaboradores realizaron un estudio transversal en 479 personas del pueblo indígena tsimane, que vive en la selva amazónica de Bolivia, bajo la hipótesis de que en las sociedades preindustriales la desigualdad en los ingresos no determina una peor salud individual (50). Esto podría deberse, como hipótesis, al elevado CS que caracteriza a estas comunidades que las protegería contra el efecto negativo que la desigualdad ejerce sobre la salud. Los autores abordaron el CS como la confianza, las redes sociales y las organizaciones comunitarias que permiten actuar colectivamente.
Como una variable indirecta de la salud, el estudio evaluó el estado nutricional de la muestra mediante el índice de masa corporal y las puntuaciones $Z$ de la suma de los pliegues cutáneos tricipital y subescapular y el área muscular braquial, estandarizadas por el sexo y la edad. Para medir la inequidad se utilizó el coeficiente de Gini de ingresos per cápita y como indicador indirecto de los ingresos brutos del año anterior se tomó el área de deforestación de cada vivienda (asociada con el consumo y la venta de bienes vinculados al terreno). Si bien en este estudio no se midió el CS, se agregó un análisis a partir de datos obtenidos entre 2002 y 2003 en 669 personas mayores de 16 años sobre los regalos hechos y el trabajo comunitario realizado, como reflejo del CS.

En el estudio no se encontró correlación entre la desigualdad en los ingresos y el estado nutricional de la población ni entre el CS y las variables antropométricas. Una posible explicación planteada por los autores es que el CS podría proteger contra el efecto deletéreo que tiene sobre el estado nutricional la desigualdad en los ingresos. Sin embargo, los resultados no son concluyentes y se deben tener en cuenta algunas limitaciones de este estudio, como la forma indirecta de medir el CS, la no inclusión del componente cognitivo del CS y el uso en la población boliviana de los parámetros antropométricos establecidos para la población estadounidense.

En otro artículo, De Silva y Harpham (51) reelaboraron los resultados del estudio internacional desarrollado en Etiopía, la India, Perú y Vietnam ya mencionado (41) con una metodología transversal cuantitativa para explorar el CS materno individual, tanto cognitivo como estructural, mediante la versión resumida del ASCAT. Para determinar la posible asociación entre el CS materno y el estado nutricional en niños de 6 a 18 meses de edad utilizaron los índices $Z$ del peso para la edad y la talla para la edad, respectivamente (según las curvas de referencia del crecimiento elaboradas por el Centro Nacional de Estadística de Salud, NCHS, de los Estados Unidos de América). En general, Perú presentó niveles de CS más bajos que los otros tres países estudiados. En el contexto peruano, solamente el recibir apoyo social de dos o más personas se asoció con una mayor talla para la edad en los niños y las niñas $(P<0,001)$, pero no con un mejor peso para la edad. Las otras áreas de CS evaluadas no mostraron corre- lación estadística significativa tras ajustar por variables de confusión.

Pese a la resumida exploración del CS lograda mediante el instrumento empleado, el hecho de haber incorporado los CS cognitivo y estructural y el haber profundizado en la validación de su instrumento representan fortalezas metodológicas de este estudio. Sin embargo, aún se trata de hallazgos preliminares. La imposibilidad de determinar la dirección causal es una limitación de esta investigación que debe considerarse al interpretar los resultados.

\section{Vacunación}

En un estudio transversal cuantitativo realizado en 482 hogares pertenecientes a seis villas de Nicaragua con $63 \%$ de población semiurbana o rural se exploró la relación entre el CS y la vacunación infantil (52). En este estudio, el CS se definió como la confianza, la reciprocidad y la participación presentes en la sociedad. Los CS cognitivo y estructural se estimaron mediante una versión adaptada del cuestionario integrado sobre el CS (Social Capital Integrated Questionnaire, SC-IQ) (53). Para la vacunación se estableció una puntuación entre 0 y 6 , en la que cada vacuna (antipoliomielítica, BCG antituberculósica, triple contra el sarampión, las paperas y la rubéola, y cada una de las tres dosis de la vacuna triple contra la difteria, el tétanos y la tos ferina) contribuía con un punto. La unidad de análisis fue el hogar.

De los elementos del CS cognitivo, la confianza social y la confianza institucional mostraron efectos opuestos. Mediante la regresión lineal se observó que una mayor confianza en la sociedad se asoció con una reducción de $50 \%$ en la probabilidad de haber recibido una vacuna adicional $(\mathrm{OR}=0,39 ; P<0,01)$, mientras que la confianza institucional se asoció con un aumento en la puntuación correspondiente a la vacunación infantil (OR = 1,7; $P<0,05)$.

Esta investigación generó una extensa evaluación del CS, sin embargo el hecho de basarse en una encuesta de más de una hora de duración en un reducido número de comunidades constituye una limitación reconocida por los autores para poder generalizar estos resultados a todo el país. Las asociaciones opuestas entre los dos tipos de CS evaluados y la salud podrían comprenderse mejor mediante metodologías cualitativas. 


\section{DISCUSIÓN}

En ALC, donde prevalece una situación de salud en evolución marcada por fuertes inequidades (54), hay conciencia de la necesidad de conocer mejor los determinantes sociales y existe un creciente interés por el desarrollo de investigaciones sobre la relación entre el CS y la salud. El aporte fundamental de la presente revisión, que complementa otro artículo previamente publicado enfocado más hacia los aspectos conceptuales y sus implicaciones en la promoción de la salud (22), es integrar los avances y los desafíos centrales para una agenda de investigación futura en ALC.

Como en toda síntesis de información (55), sistematizar esta búsqueda permitió minimizar eventuales sesgos en esta área; sin embargo, la exclusión de información no publicada limitó el alcance de las conclusiones. Una gran cantidad de estudios no llegan a publicarse, en parte por no encontrar relaciones estadísticamente significativas (56), lo que puede haber provocado un importante sesgo al seleccionar las publicaciones a analizar, algo que adquiere mayor importancia por no existir aún una certidumbre total sobre el papel que desempeña el CS en la salud. Lo incipiente del desarrollo local en este tema y la amplia gama de resultados de salud que exploraban los estudios analizados obligó a emplear una pregunta guía necesariamente amplia para cubrir los objetivos de esta revisión. Esto impidió profundizar más en los detalles específicos de cada variable de salud en particular. Además, la diversidad en las metodologías utilizadas para estudiar el CS, enfocadas hacia diferentes unidades de análisis (individual, ecológico o mixto) dificultó considerablemente la comparación e integración de los resultados. Todo esto hizo más difícil lograr una síntesis general más concluyente sobre la relación entre el CS y la salud en ALC, aunque en determinadas áreas fue posible aproximarse a conclusiones preliminares.

La evidencia actual no permite confirmar —aunque sí esbozar- la relación entre el CS y la salud en ALC. Los resultados analizados indican que un mayor CS en una población se asociaría con una mejor salud mental y un menor riesgo de trauma dental por accidentes. La evidencia es menos clara y menos directa en cuanto a la mortalidad, la esperanza de vida y el estado nutricional.
Si bien el papel protector que tendría el CS cognitivo sobre la depresión y la ansiedad, incluso en diferentes contextos socioculturales, surge de estudios realizados en poblaciones específicas (jóvenes y madres), todo parece indicar que la salud mental es el área donde los resultados son más claros, ya que se exploran unidades clínicas definidas y su relación con el CS mediante metodologías comparables. Varios autores han informado resultados similares $(57,58)$ y han identificado la cohesión social y la confianza como los únicos elementos del CS que se asociarían con el bienestar psicológico (58). Además, este resultado se logró mediante el cuestionario general de salud (59), que es un instrumento ampliamente aceptado como herramienta de tamizaje (60). De esta manera, si bien se podría plantear que la confianza social ejerce un papel protector transcultural sobre la salud mental, es importante aclarar que los diseños metodológicos actuales no permiten establecer una dirección causal en esta relación y que al medir el CS no se puede descartar el efecto negativo del sesgo de respuesta debido al estado psicológico del entrevistado, lo que hace necesario realizar más investigaciones en este campo. Tampoco es posible formular conclusiones sólidas respecto a la relación entre el consumo de bebidas alcohólicas y el CS.

El apoyo social, como posible factor asociado con la talla infantil, pudiera ser importante como hallazgo preliminar para investigaciones futuras. Un estudio realizado en madres puertorriqueñas residentes en los Estados Unidos (61) encontró una fuerte asociación entre la lactancia materna, por una parte, y el intercambio de apoyo y los servicios entre los familiares y los vecinos, por la otra, lo que reafirmó la relevancia que el CS podría tener en el desarrollo y la salud de los niños y las niñas (62).

Para lograr una mejor comprensión de la eventual relación entre el CS y la salud en ALC, y tomando en cuenta que el CS depende de aspectos cognitivos y relacionales propios de cada cultura, se deben emprender estudios cualitativos que permitan enriquecer los resultados cuantitativos o ayuden a comprenderlos. Las variaciones encontradas en cuanto al efecto de la confianza social e institucional sobre la salud podrían ser el reflejo de los diferentes efectos que determinados subcomponentes del CS ejercen sobre la salud en dependencia del con- texto sociocultural en el que se den, como indican los resultados de la vacunación infantil en Nicaragua (52). Por ejemplo, cuando una persona deposita mayor confianza en su comunidad, los efectos en salud podrían ser negativos si la comunidad no provee consejos de salud adecuados.

Entre las principales limitaciones de los estudios encontrados se deben destacar: a) se emplearon diversas definiciones de CS y los resultados de salud evaluados no siempre se expusieron de forma clara; b) no siempre se emplearon instrumentos adecuadamente validados en el contexto de ALC para evaluar el CS; c) algunas particularidades y la diversidad de las poblaciones evaluadas no permiten hacer generalizaciones; d) los pequeños tamaños de muestra empleados en muchos de los estudios resultaron insuficientes para detectar efectos menores sobre la salud; e) la falacia ecológica y las propias limitaciones de los estudios transversales impidieron alcanzar resultados más robustos. Se debe señalar que muchas de estas limitaciones son también propias de estudios sobre el CS en otros contextos culturales, como Europa y los Estados Unidos.

Urge desarrollar y sistematizar investigaciones sobre los determinantes sociales de la salud en ALC. Para evaluar la relación entre el CS y la salud es fundamental profundizar en la validación de instrumentos de medición, como los presentados por De Silva y colaboradores (23), adecuados para las características culturales de cada país. También se deben emprender estudios longitudinales que permitan establecer la posible dirección causal de la relación entre el CS y la salud. La complementariedad de las metodologías cualitativas y cuantitativas puede ser de gran ayuda para comprender mejor esta relación en cada contexto particular. Del mismo modo, es imperativo alcanzar consensos para articular y potenciar la investigación entre los países, generar redes y, eventualmente, estudios multicéntricos.

No se debe perder de vista la importancia que pudiera tener el CS como factor facilitador de los procesos colectivos en favor de la salud y las intervenciones asociadas (63). Por ejemplo, los consejos de salud en Brasil han contribuido a la activación y el crecimiento de organizaciones vecinales y agrupaciones de portadores de algunas enfermedades y han servido para encaminar algunas deman- 
das por más y mejor asistencia médica (64). Intervenciones como esta sirven para involucrar a la sociedad civil en el mejoramiento de la salud; sin embargo, si no se acompañan con una adecuada gestión se corre el riesgo de generar frustración en la comunidad por el bajo retorno que tienen sus iniciativas (64). En el mediano plazo se requiere evaluar, mediante investigaciones cuasiexperimentales $\mathrm{u}$ otros diseños convenientes, el impacto que puede tener la incorporación del CS en las intervenciones de salud -de forma explícita y adecuada al contexto- y determinar cómo esto puede influir en los resultados sobre la salud de la población.

Finalmente, se deben tener en cuenta las propias limitaciones del concepto de CS, desde la propia ambigüedad y varia- bilidad de las definiciones hasta los riesgos que implica la simplificación excesiva de sus posibilidades y efectos. Más allá de estas limitaciones, el desafío de hoy es poder valorar y considerar la importancia real de los determinantes sociales en el diseño, la implementación y la evaluación de los programas y las acciones tendientes a alcanzar una mayor equidad en salud en ALC. La investigación, aún incipiente en esta área, deberá desempeñar un papel central y apoyar la toma de decisiones más adecuadas y la implementación de políticas pertinentes, con la urgencia y la calidad necesarias.

A pesar de las limitaciones propias del concepto de CS y de los estudios identificados, se puede afirmar que existe evidencia científica incipiente de la posible relación entre el CS y la salud en ALC.
El CS podría desempeñar un papel protector en ciertas áreas sanitarias, como la prevención de traumas y la salud mental; no obstante, la relación entre el CS y la salud podría variar según los subtipos de CS y los contextos socioeconómicos y culturales específicos. Resulta fundamental continuar desarrollando investigaciones en el ámbito de la epidemiología social, en las que se analice en profundidad la relación entre los determinantes sociales y los aspectos específicos de la salud en el contexto de ALC.

Agradecimientos. Con esta revisión se da continuidad a una línea de investigación sobre capital social y salud en la comunidad en Chile que está relacionada con una iniciativa previa (Proyecto FONDECYT \#1050709/2005).

\section{REFERENCIAS}

1. Krieger N. A glossary for social epidemiology. J Epidemiol Community Health. 2001;55: 693-700.

2. Tarlov A. Social determinants of health: the sociobiological translation. In: Blane D, Brunner E, Wilkinson R, eds. Health and social organization. London: Routledge; 1996. Pp. 71-93.

3. Wilkinson R, Marmot M, eds. Social determinants of health: the solid facts. Copenhagen: WHO; 2003.

4. Fukuyama F. Trust: the social virtues and the creation of prosperity. New York: Free Press; 1995.

5. Sampson RJ, Raudenbush SW, Earls F. Neighborhoods and violent crime: a multilevel study of collective efficacy. Science. 1997;277: 918-24.

6. Field J. Social capital and lifelong learning. Bristol: Policy Press; 2005.

7. Lin N. Social capital. A theory of social structure and action. Cambridge: Cambridge University Press; 2001.

8. Atria R, Siles M. Capital social y reducción de la pobreza: en busca de un nuevo paradigma. Santiago: Universidad de Michigan, CEPAL; 2003.

9. Bourdieu P, Loïc W. An invitation to reflexive sociology. Chicago: University of Chicago Press; 1992.

10. Coleman J. Foundations of social theory. Cambridge (MA): Harvard University Press; 1990.

11. Putnam R, Leonardi R, Nanetti RY. Making democracy work: civic traditions in modern Italy. Princeton, NJ: Princeton University Press; 1994.

12. Portes A. Social capital: its origins and applications in modern sociology. Ann Rev Sociol. 1998;24:1-24.

13. Islam KM, Merlo J, Kawachi I, Lindström M, Gerdtham U. Social capital and health: does egalitarianism matter? A literature review. Int J Equity Health. 2006;5:3.
14. Lomas J. Social capital and health: implications for public health and epidemiology. Soc Sci Med. 1998;47:1181-8.

15. Kim D, Subramanian SV, Kawachi I. Bonding versus bridging social capital and their associations with self rated health: a multilevel analysis of 40 US communities. J Epidemiol Community Health. 2006;60(2):116-22.

16. Petrou S, Kupek E. Social capital and its relationship with measures of health status: evidence from the Health Survey for England 2003. Health Econ. 2008;14(1):127-43.

17. Nummela $O$, Sulander $T$, Rahkonen $O$, Karisto A, Uutela A. Social participation, trust and self-rated health: a study among ageing people in urban, semi-urban and rural settings. Health Place. 2008;14(2): 243-53.

18. Lindström C, Lindström M. "Social capital," GNP per capita, relative income, and health: an ecological study of 23 countries. Int J Health Serv. 2006;36(4):679-96.

19. Muntaner C. Commentary: social capital, social class, and the slow progress of psychosocial epidemiology. Int J Epidemiol. 2004;33:674-80.

20. Carpiano RM. Neighborhood social capital and adult health: an empirical test of a Bourdieu-based model. Health Place. 2007;13(3): 639-55.

21. Pattussi MP, Moysés SJ, Junges JR, Sheiham A. Capital social e a agenda de pesquisa em epidemiologia. Cad Saude Publica. 2006;22: 1525-46.

22. Sapag J, Kawachi I. Social capital and health promotion in Latin America. Rev Saude Publica. 2007;41(1):139-49.

23. De Silva MJ, Harpham T, Tuan T, Bartolini R, Penny ME, Huttly SR. Psychometric and cognitive validation of a social capital measurement tool in Peru and Vietnam. Soc Sci Med. 2006;62(4):941-53.

24. Idrovo AJ. Income inequality, corruption, and life expectancy at birth in Mexico. Rev. Salud Publica. 2005;7(2):121-9.
25. Idrovo AJ. Social capital, violent deaths, and cancer mortality in Colombia: a population approach. Rev Salud Publica. 2006;8(1):38-51.

26. Muntaner C, Lynch J. Income inequality, social cohesion, and class relations: a critique of Wilkinson's neo-Durkheimian research program. Int J Health Serv. 1999;29: 59-81.

27. García-Sucre M, Bunge M. Differentiation, participation and cohesion. Qual Quant. 1989; 34:697-703.

28. Martikainen $\mathrm{P}$, Kauppinen TM, Valkonen T. Effects of the characteristics of neighborhoods and the characteristics of people on cause specific mortality: a register based follow up study of 252,000 men. J Epidemiol Community Health. 2003;57:210-7.

29. Harpham T, Grant E, Rodríguez C. Mental health and social capital in Cali, Colombia. Soc Sci Med. 2004;58(11):2267-77.

30. Harpham T, Snoxell S, Grant E, Rodríguez C. Common mental disorders in a young urban population in Colombia. Br J Psychiatry. 2005;187:161-7.

31. Harpham T, Grant E, Thomas E. Measuring social capital in health surveys: key issues. Health Policy Plan. 2002;17(1):106-11.

32. World Health Organization. The world health report: mental health. Geneva: WHO; 2001.

33. Orpinas P. Progress report of the multi-centre project on violence (Project ACTIVA). Washington, D.C.: Pan American Health Organization; 1998.

34. Ewing JA. Detecting alcoholism. The CAGE questionnaire. J Am Med Assoc. 1984;252(14): 1905-7.

35. Rosenheck R, Morrisey J, Lam J, Calloway M, Stolar M, Johnsen M, et al. Service delivery and community: social capital, service systems integration and outcomes among homeless person with severe mental illness. Health Serv Res. 2001;36:691-710. 
36. Lundborg P. Social capital and substance use among Swedish adolescents: an explorative study. Soc Sci Med. 2005;61(6):1151-8

37. Weitzman ER, Kawachi I. Giving means receiving: the protective effect of social capital on binge drinking on college campuses. Am J Public Health. 2000;90(12):1936-9.

38. Godoy RA, Reyes-García V, McDade T, Huanca T, Leonard WR, Tanner S, et al. Does village inequality in modern income harm the psyche? Anger, fear, sadness, and alcohol consumption in a pre-industrial society. Soc Sci Med. 2006:63(2):359-72.

39. Rose R. How much does social capital add to individual health? A survey study of Russians. Soc Sci Med. 2000;51(9):1421-35.

40. Ekholm O. Influence of the recall period on self-reported alcohol intake. Eur J Clin Nutr. 2004;58(1):60-3.

41. De Silva MJ, Huttly SR, Harpham T, Kenward MG. Social capital and mental health: a comparative analysis of four low income countries. Soc Sci Med. 2007;64:5-20.

42. Young Lives Project. Young Lives: an international study of childhood poverty. Research methodology and data. Oxford: University of Oxford, Young Lives Project. Hallado en http:/ / www.younglives.org.uk/ researchmethodology-data. Acceso el 1 de febrero de 2009.

43. World Health Organization. A user's guide to the self-reporting questionnaire (SRQ). Geneva: WHO; 1994.

44. Pattussi MP, Hardy R, Sheiham A. Neighborhood social capital and dental injuries in Brazilian adolescents. Am J Public Health. 2006; 96(8):1462-8.

45. Diez-Roux AV. Investigating neighborhood and area effects on health. Am J Public Health. 2001;91:1783-9.

46. Inclán $\mathrm{C}$, Hijar $\mathrm{M}$, Tovar V. Social capital in settings with a high concentration of road traffic injuries. The case of Cuernavaca, Mexico. Soc Sci Med. 2005;61(9):2007-17.

47. Burström B. Social differentials in the decline of infant mortality in Sweden in the twentieth century: the impact of politics and policy. Int J Health Serv. 2003;33:723-41.

48. Magalhães R. Monitoring inequalities in health: meanings and strengths of information sources. Cienc Saude Coletiva. 2007; 12(3): $667-73$.

49. Pearce N, Davey Smith G. Is social capital the key to inequalities in health? Am J Public Health. 2003;93(1):122-9.

50. Godoy R, Byron E, Reyes-García V, Valdez V, Leonard WR, Apaza L, et al. Income inequality and adult nutritional status: anthropometric evidence from a pre-industrial society in the Bolivian Amazon. Soc Sci Med. 2005;61(5): 907-19.

51. De Silva MJ, Harpham T. Maternal social capital and child nutritional status in four developing countries. Health Place. 2007;13(2): 341-55.

52. Mitchell AD, Bossert TJ. Measuring dimensions of social capital: evidence from surveys in poor communities in Nicaragua. Soc Sci Med. 2007:64(1):50-63.

53. Grootaert C, Narayan D, Jones VN, Woolcock. Integrated questionnaire for the measurement of social capital. Washington, D.C.: World Bank; 2003.

54. Dachs J, Norberto W, Ferrer M, Florez CE, Barros AJD, Narváez R, et al. Inequalities in health in Latin America and the Caribbean: descriptive and exploratory results for selfreported health problems and health care in twelve countries. Rev Panam Salud Publica. 2002;11 (5-6):335-55.

55. Oxman AD, Cook DJ, Guyatt GH. User's guides to the medical literature. VI. How to use an overview. J Am Med Assoc. 1994; 272(17):1367-71.
56. Montori VM, Smieja M, Guyatt GH. Publication bias: a brief review for clinicians. Mayo Clin Proc. 2000;75:1284-8.

57. Poblete F, Sapag J, Bossert T. Capital social y salud mental en comunidades urbanas de nivel socioeconómico bajo en Santiago, Chile. Nuevas formas de entender la relación comunidad-salud. Rev Med Chile. 2008;136:230-9.

58. Araya R, Dunstan F, Playle R, Thomas H, Palmer S, Lewis G. Perceptions of social capital and the built environment and mental health. Soc Sci Med. 2006;62(12):3072-83.

59. Goldberg D, Williams P. The user's guide to the general health questionnaire. Windsor, England: NFER-Nelson; 1988.

60. Berardi D, Berti Ceroni G, Leggieri G, Rucci P, Ustun B, Ferrari G. Mental, physical and functional status in primary care attenders. Int J Psychiatry Med. 1999;29(2):133-48.

61. Anderson AK, Himmelgreen DA, Peng YK, Segura-Pérez S, Pérez-Escamilla R. Social capital and breastfeeding initiation among Puerto Rican women. Adv Exp Med Biol. 2004;554:283-6.

62. Janssens W, Gaag JVD, Gunning JW. The role of social capital in early childhood development: evidence from rural India. Amsterdam: Institute for International Development; 2005.

63. Pridmore P, Thomas L, Havemann K, Sapag J, Wood L. Social capital and healthy urbanization in a globalized world. J Urban Health. 2007;84(3 Suppl):i130-43.

64. Labra ME. Social capital and health councils in Brazil: a virtuous circle? Cad Saude Publica. 2002;18(Suppl):47-55.

Manuscrito recibido el 27 de octubre de 2007. Aceptado para publicación, tras revisión, el 2 de junio de 2008.

ABSTRACT Objective. To identify validated information available on the relationship between social capital (SC) and health in Latin America and the Caribbean (LAC).

\section{Social capital and health in Latin America and the Caribbean: a systematic review}

Methods. A systematic search for papers published from January 1990-June 2007 was conducted on the Medline, SciELO, LILACS, and the Cochrane Library databases. All of the research and review articles published by scientific journals that evaluated CS and its relationship to health in LAC were included in the analysis.

Results. Fifteen articles were found (11 original research and 4 reviews). The health topics examined by the research articles were: (a) mortality and life expectancy, (b) mental health, (c) injuries, (d) nutritional status, and (e) immunization. The validity of these works, the results, and the principal conclusions were analyzed in depth.

Conclusions. Despite limitations inherent to the CS concept and the studies identified, it was confirmed that scientific evidence exists to support the possible association between CS and health in LAC. CS could play a protective role in certain health areas, such as injury prevention and mental health; not withstanding, the relationship between CS and health could vary according to CS subtype and socioeconomic context and culture. It is therefore imperative to continue social epidemiology research that thoroughly investigates the relationship between social determinants and specific aspects of health in LAC context.

Key words Social participation, health, Latin America, Caribbean Region. 